\title{
A Generalisation of Jacobi's Fundamental Formulae
}

\author{
By J. M. RUSHFORTH
}

(Received I1th July, 1949. Read 4th November, 1949.)

1. Jacobi obtained his well-known formulae by a purely algebraic method ${ }^{1}$, but it was not until H. J. S. Smith had obtained them similarly ${ }^{2}$, but by the use of a more symmetrical notation, that they were put into the form by which they are known today.

The basic formula is

$$
\begin{aligned}
& \begin{array}{l}
2 \vartheta_{3}(w) \vartheta_{3}(x) \vartheta_{3}(y) \vartheta_{3}(z) \\
=\vartheta_{3}\left(w^{\prime}\right) \vartheta_{3}\left(x^{\prime}\right) \vartheta_{3}\left(y^{\prime}\right) \vartheta_{3}\left(z^{\prime}\right)+\vartheta_{4}\left(w^{\prime}\right) \vartheta_{4}\left(x^{\prime}\right) \vartheta_{4}\left(y^{\prime}\right) \vartheta_{4}\left(z^{\prime}\right) \\
\quad+\vartheta_{2}\left(w^{\prime}\right) \vartheta_{2}\left(x^{\prime}\right) \vartheta_{2}\left(y^{\prime}\right) \vartheta_{2}\left(z^{\prime}\right)-\vartheta_{1}\left(w^{\prime}\right) \vartheta_{1}\left(x^{\prime}\right) \vartheta_{1}\left(y^{\prime}\right) \vartheta_{1}\left(z^{\prime}\right)
\end{array}
\end{aligned}
$$

where

$$
\begin{aligned}
& 2 w^{\prime}=-w+x+y+z, \\
& 2 x^{\prime}=w-x+y+z, \\
& 2 y^{\prime}=w+x-y+z, \\
& 2 z^{\prime}=w+x+y-z .
\end{aligned}
$$

The other formulae may be obtained from this by the addition of suitable half periods to the variables.

The formulae in this paper are obtained by a generalisation of Smith's method and, so far as I am aware, they have not been given previously. They relate a product of $2 \mathrm{~N}$ theta-functions in one set of variables to a sum of $N^{2}$ terms in the other set, each term containing a product of $2 N$ theta-functions. For the general case, with $N>2$, they do not have such a wide range of application as Jacobi's formulae, but nevertheless they possess an elegance which makes them worth recording.

2. To simplify the notation we will adopt the following conventions throughout. We suppose that $N$ is a positive integer, and we write the sum

$$
x_{1}+x_{2}+\ldots+x_{2 N}=\sum_{j=1}^{2 N} x_{j}
$$

and omit the limits of summation when no risk of confusion occurs. We also write similar expressions in $m, m^{2}, n, n^{2}, m x, n X$ as $\Sigma m_{j}, \Sigma m_{j}{ }^{2}, \Sigma n_{j}$,

1 C. G. J. Jacobi, Ges. Werke, I, pp. 502-6.

2 H. J. S. Smith, Proc. London Math. Soc., I (1866), 1-12. 
$\sum n_{j}{ }^{2}, \sum m_{j} x_{j}, \sum n_{j} X_{j}$. A product of $2 N$ terms of this kind is indicated in the same manner, for instance $\prod_{j=1}^{2 N} \vartheta_{3}\left(x_{j}\right)$ is written as $\Pi \vartheta_{3}\left(x_{j}\right)$.

3. Consider the set of equations

$$
\begin{aligned}
& N\left(X_{i}+x_{i}\right)=\Sigma x_{j}, \\
& N\left(n_{i}+m_{i}\right)=\Sigma m_{j},
\end{aligned}
$$

where $i$ runs from 1 to $2 N$.

It will be seen immediately that

$$
\sum n_{j}=\Sigma m_{j}, \quad \sum n_{j}^{2}=\Sigma m_{j}^{2}, \quad \Sigma n_{j} X_{j}=\Sigma m_{j} x_{j} .
$$

We now take the product of $2 N \vartheta_{3} \mathrm{~s}$, expand them in series and use the relations we have just obtained. We get

$$
\begin{aligned}
\Pi \vartheta_{3}\left(x_{j}\right) & =\sum_{n_{j}=-\infty}^{\infty} \ldots \sum(j=1,2, \ldots, 2 N) \\
& =\sum_{m_{j}(j=1,2, \ldots, 2 N)}^{\sum \ldots \Sigma} q^{\Sigma m_{j}{ }^{2}} e^{\Sigma m_{j} x_{j}} .
\end{aligned}
$$

The summation in this last term will be over all the values of $m_{j}$ corresponding to the values of $n_{j}$ and, from (2), these will be of the form: an integer plus $\left(\Sigma m_{j}\right) / N$. We separate this sum into $N$ sets of terms $S_{r}$, where $r=0,1,2, \ldots, N-1$, in which $\Sigma m_{j} \equiv r(\bmod N)$.

Then

$$
\Pi \vartheta_{3}\left(X_{j}\right)=\sum_{r=0}^{N-1} S_{r}
$$

and

$$
S_{r}=\sum_{m_{j}(j=1,2, \ldots, 2 N)} q^{\Sigma m_{j}} e^{2 i \Sigma m_{j} x_{j}},
$$

which is summed over all $m_{j}$ such that $m_{j}=$ an integer plus $r / N$ and $\sum m_{j} \equiv r(\bmod N)$.

We can introduce a factor

$$
\frac{1}{N} \sum_{s=0}^{N-1} e^{2 \pi i\left(\Sigma m_{j}-r\right) s / N}
$$

into $S_{r}$, and sum over all $m_{j}$ equal to an integer plus $r / N$, since this factor is unity if $\Sigma m_{j} \equiv r(\bmod N)$, and is the sum of the $N$-th roots of unity if $\sum m_{j} \neq \equiv r(\bmod N)$.

We write then

$$
S_{r}=\frac{1}{N} \sum_{s=0}^{N-1} \sum_{l_{j}=-\infty}^{\infty} \underset{\left(m_{j}=l_{j}+r / N\right)}{\sum} e^{2 \pi i\left(\Sigma m_{j}-r\right) \& / N} q^{\Sigma m_{j}{ }^{2}} e^{2 i \Sigma m_{j} x_{j}}
$$

In this formula, and in what follows, the $l_{j}$ are integers corresponding to the integer parts of the $m_{j}$, and they are summed from $-\infty$ to $+\infty$. 
Putting $m_{j}=l_{j}+r / N$ we get

$$
\begin{aligned}
& S_{r}=\frac{1}{N} \sum_{s=0}^{N-1} e^{-2 \pi i r s / N} \sum_{l j=-\infty}^{\infty} \ldots \sum_{(j=1,2, \ldots, 2 N)} e^{(2 \pi i s / N) . \Sigma\left(l_{j}+r / N\right)} q^{\Sigma\left(l_{j}+r / N\right)^{2}} \\
& \times \exp \left\{2 i \Sigma\left(l_{j}+r / N\right) x_{j}\right\} .
\end{aligned}
$$

Remembering that $q=e^{\text {rir }}$ we can write this as

$\frac{1}{N} \sum_{s=0}^{N-1} e^{-2 \pi i r s / N} \Pi e^{2 i r r_{j} / N} e^{2 \pi i r \varepsilon / N^{2}} q^{\tau^{2} / N^{2}} \sum_{l_{j}=-\infty}^{\infty} q^{l^{2}} \exp \left\{2 i l_{j}\left(x_{j}+\frac{\pi s}{N}+\frac{\pi r \tau}{N}\right)\right\}$

$$
\begin{aligned}
& =\frac{1}{N} \sum_{s=0}^{N-1} e^{-2 \pi i r s / N} \Pi e^{2 i r x_{j} / N} e^{2 \pi i r s / N^{2}} q^{r^{2} / N^{2}} \vartheta_{3}\left(x_{j}+\frac{\pi s}{N}+\frac{\pi r \tau}{N}\right) \\
& =\frac{1}{N} \sum_{s=0}^{N-1} e^{-2 \pi i r s / N} \Pi \exp \left\{\frac{2 i r}{N}\left(x_{j}+\frac{\pi s}{N}+\frac{\pi r \tau}{N}\right)\right\} q^{-r^{2} / N^{2}} \vartheta_{3}\left(x_{j}+\frac{\pi s}{N}+\frac{\pi r \tau}{N}\right) .
\end{aligned}
$$

Our relation becomes finally

$$
\begin{aligned}
& N \prod_{j=1}^{2 N} \vartheta_{3}\left(X_{j}\right) \\
& \quad=\sum_{r, s,=0}^{N-1} e^{-2 \pi i r s / N} \prod_{j=1}^{2 N} \exp \left\{\frac{2 i r}{N}\left(x_{j}+\frac{\pi s}{N}+\frac{\pi r \tau}{N}\right)\right\} q^{-r^{2} / N^{2}} \vartheta_{3}\left(x_{j}+\frac{\pi s}{N}+\frac{\pi r \tau}{N}\right) .
\end{aligned}
$$

Three other formulae are immediately derived from this by increasing all the $X_{j}$ by $\frac{1}{2} \pi, \frac{1}{2} \pi \tau, \frac{1}{2} \pi+\frac{1}{2} \pi \tau$, so that the $x_{j}$ are also increased by $\frac{1}{2} \pi, \frac{1}{2} \pi \tau$, $\frac{1}{2} \pi+\frac{1}{2} \pi \tau$.

These formulae are

$$
\begin{aligned}
& N \prod_{j=1}^{2 N} \vartheta_{4}\left(X_{j}\right) \\
& \quad=\sum_{r, 8=0}^{N-1} e^{-2 \pi i r s / N} \prod_{j=1}^{2 N} \exp \left\{\frac{2 i r}{N}\left(x_{j}+\frac{\pi s}{N}+\frac{\pi r \tau}{N}\right)\right\} q^{-r^{2} / N^{2}} \vartheta_{4}\left(x_{j}+\frac{\pi s}{N}+\frac{\pi r \tau}{N}\right),
\end{aligned}
$$

$$
N \prod_{j=1}^{2 N} \vartheta_{2}\left(X_{j}\right)
$$$$
=\sum_{r, 8=0}^{N-1} e^{-2 \pi i r s / N} \prod_{j=1}^{2 N} \exp \left\{\frac{2 i r}{N}\left(x_{j}+\frac{\pi s}{N}+\frac{\pi r \tau}{N}\right)\right\} q^{r(N-r) / N^{2}} \vartheta_{2}\left(x_{j}+\frac{\pi s}{N}+\frac{\pi r \tau}{N}\right),
$$

$N \prod_{j=1}^{2 N} \vartheta_{1}\left(X_{j}\right)$

$$
=\sum_{r, s=0}^{N-1} e^{-2 \pi i r s / N} \prod_{j=1}^{2 N} \exp \left\{\frac{2 i r}{N}\left(x_{j}+\frac{\pi s}{N}+\frac{\pi r \tau}{N}\right) q_{j}^{\prime} q^{r(N-r) / N^{2}} \vartheta_{1}\left(x_{j}+\frac{\pi s}{N}+\frac{\pi r \tau}{N}\right) .\right.
$$

\section{Department of Pure Mathematics,}

The University,

\section{Birmingham.}

